\title{
DECIMER: towards deep learning for chemical image recognition
}

\author{
Kohulan Rajan ${ }^{1}$, Achim Zielesny ${ }^{2}$ and Christoph Steinbeck ${ }^{1 *}$ (D)
}

\begin{abstract}
The automatic recognition of chemical structure diagrams from the literature is an indispensable component of workflows to re-discover information about chemicals and to make it available in open-access databases. Here we report preliminary findings in our development of Deep IEarning for Chemical ImagE Recognition (DECIMER), a deep learning method based on existing show-and-tell deep neural networks, which makes very few assumptions about the structure of the underlying problem. It translates a bitmap image of a molecule, as found in publications, into a SMILES. The training state reported here does not yet rival the performance of existing traditional approaches, but we present evidence that our method will reach a comparable detection power with sufficient training time. Training success of DECIMER depends on the input data representation: DeepSMILES are superior over SMILES and we have a preliminary indication that the recently reported SELFIES outperform DeepSMILES. An extrapolation of our results towards larger training data sizes suggests that we might be able to achieve near-accurate prediction with 50 to 100 million training structures. This work is entirely based on open-source software and open data and is available to the general public for any purpose.
\end{abstract}

Keywords: Optical chemical entity recognition, Chemical structure, Deep learning, Deep neural networks, Autoencoder/decoder

\section{Main text}

The automatic recognition of chemical structure diagrams from the chemical literature (herein termed Optical Chemical Entity Recognition, OCER) is an indispensable component of workflows to re-discover information about chemicals and to make it available in open-access databases. While the chemical structure is often at the heart of the findings reported in chemical articles, further information about the structure is present either in textual form or in other types of diagrams such as titration curves, spectra, etc. (Fig. 1).

Previous software systems for OCER have been described and were both incorporated into commercial and open-source systems. These software systems

\footnotetext{
*Correspondence: christoph.steinbeck@uni-jena.de

${ }^{1}$ Institute for Inorganic and Analytical Chemistry, Friedrich-SchillerUniversity Jena, Lessingstr. 8, 07743 Jena, Germany

Full list of author information is available at the end of the article
}

include Kekulé [1, 2], the Contreras system [3], the IBM system [4], CLIDE [5] as well as the open-source approaches chemOCR [6-8], ChemReader [9], OSRA [10] and ChemRobot described in a patent [11].

All of these software packages share a general approach to the problem, comprising the steps (a) scanning, (b) vectorization, (c) searching for dashed lines and dashed wedges, (d) character recognition, (e) graph compilation, (f) post-processing, (g) display and editing.

Each of the steps in such systems needs to be carefully hand-tuned both individually as well as for its interplay with the other steps. The incorporation of new image features to be detected is a laborious process.

We were recently inspired by the stunning success of AlphaGo Zero [13], a deep neural network (NN) based approach that enabled AlphaGo Zero to reach superhuman strength in the Game of Go by playing a potentially unlimited number of games against itself, starting with

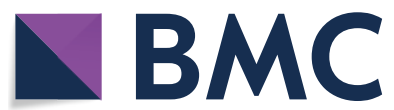

(c) The Author(s) 2020. This article is licensed under a Creative Commons Attribution 4.0 International License, which permits use, sharing, adaptation, distribution and reproduction in any medium or format, as long as you give appropriate credit to the original author(s) and the source, provide a link to the Creative Commons licence, and indicate if changes were made. The images or other third party material in this article are included in the article's Creative Commons licence, unless indicated otherwise in a credit line to the material. If material is not included in the article's Creative Commons licence and your intended use is not permitted by statutory regulation or exceeds the permitted use, you will need to obtain permission directly from the copyright holder. To view a copy of this licence, visit http://creativeco mmons.org/licenses/by/4.0/. The Creative Commons Public Domain Dedication waiver (http://creativecommons.org/publicdomain/ zero/1.0/) applies to the data made available in this article, unless otherwise stated in a credit line to the data. 


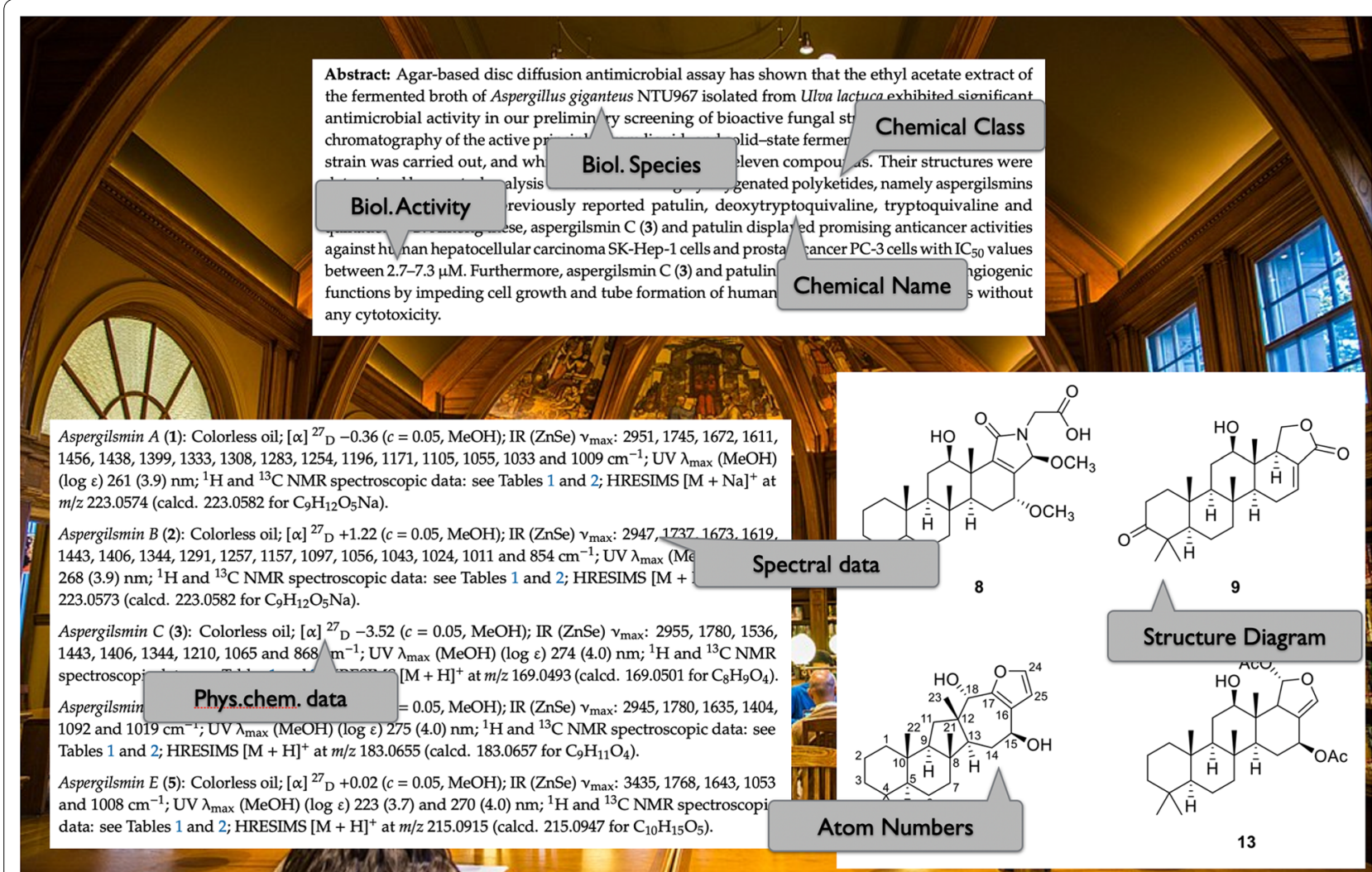

Fig. 1 Information about a natural product is scattered across the various sections of an individual scientific article. Grouped around a structure and a chemical name, further information such as chemical classes, species, and organism parts from which the compound was isolated, spectral and other data are listed. Background image @ Alina Chan, distributed under https://creativecommons.org/licenses/by-sa/4.0/deed.en, figures and text from Kwon et al. [12]

no more knowledge than the basic rules of the game. In this example, as well as in other prominent examples of successful deep learning, the key to success was the availability of a potentially unlimited or very large amount of training data.

The example of AlphaGo Zero made us realize that we are in a similar situation for the visual computing challenge described above. Instead of working with a necessarily small corpus of human-annotated examples from the printed literature, as has been common in the text mining and machine learning applications in chemistry in the past, we realised that we could generate training data from a practically unlimited source of structures generated by structure generators or by using the largest collections of open chemical data available to mankind.

After we started our work presented here, other attempts to use deep learning for OCER were reported. Work by the Schrödinger group [14] reports the successful extraction of machine-readable chemical structures from bitmaps but no software system available for the general public to replicate the reported results. A method called Chemgrapher [15] suggests to deal with the problem in a modular fashion by using a segmentation algorithm to segment the images containing chemical graphs to detect atoms locations, bonds and charges separately, and employ a graph building algorithm to re-generate the chemical graph.

Here we report preliminary findings of our development of Deep lEarning for Chemical ImagE Recognition (DECIMER), a deep learning method based on existing show-and-tell deep neural networks, which translates a pure bitmap image of a molecule, as found in publications, into a SMILES (Fig. 2). Unlike for example Chemgrapher, it makes no prior assumptions, such as the existence of bonds or element symbols in the graphic, about the structure of the underlying problem.

The training state reported here does not yet rival the performance of existing traditional approaches, but we present evidence that, given sufficient training data, our method will reach a comparable detection power without 


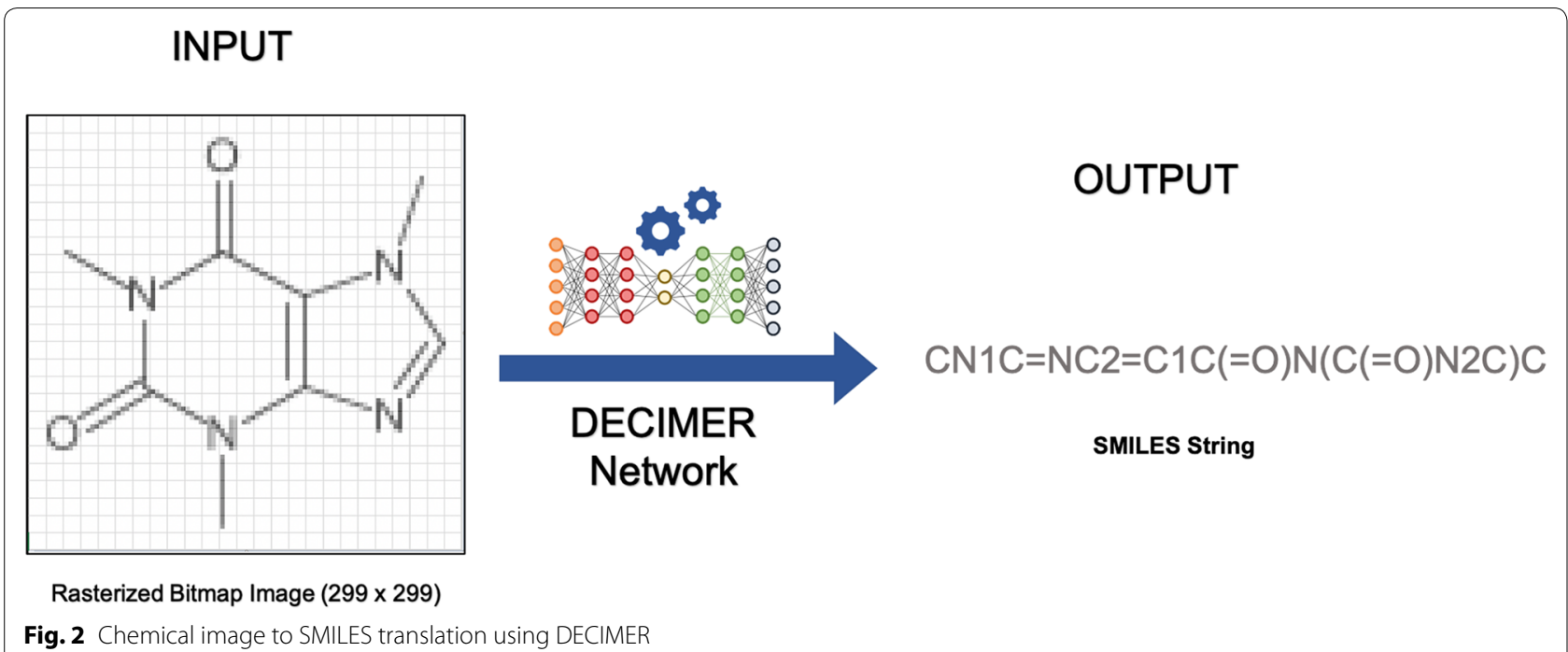

the need of the sophisticated engineering steps of an OCER workflow.

The principal idea reported here is to repurpose a show-and-tell deep NN designed for general photo annotation earlier and train it to report a series of SMILES tokens when presented with the bitmap of a chemical structure image. The original NN reported sentences like "A giraffe standing in a forest with trees in the background" when presented with a corresponding photo.

Instead of abstracting chemical diagrams from the chemical literature to generate training data, we decided to use structure diagram generators (SDG) like the one found in the Chemistry Development Kit (CDK) [16] to generate a potentially unlimited amount of training data. This type of training data can be accommodated to become more realistic and comparable to the varying picture quality in the chemical literature by using image manipulation such as blurring, adding noise, etc. As a source of input structures for the CDK SDG, we turned to PubChem [17], one of the largest databases of organic molecules. The following rules were used to curate the Pubchem data for our work presented here (in future versions of this deep $\mathrm{NN}$, these rules might be relaxed):

- Molecules must,

- Have a molecular weight of fewer than 1500 Daltons,

- Not possess counter ions,

- Only contain the elements C, H, O, N, P, S, F, Cl, Br, I, Se and B,
- Not contain isotopes of Hydrogens (D, T),

- Have 5-40 bonds,

- Not contain any charged groups,

- Only contain implicit hydrogens, except in functional groups,

- Have less than 40 SMILES characters.

The generation of molecular bitmap images from chemical graphs was performed using the CDK SDG, which generates production quality 2D depictions to feed the deep learning algorithm. One random rotation for each molecule was used. No further modifications, such as the addition of noise, were applied. These types of modifications will be explored once a mature model has been reached.

The text data used here were SMILES [18] strings, which were encoded into different formats, regular SMILES, DeepSMILES [19] and SELFIES [20], to test the dependency of the learning success on the data representation. These datasets were used in different training models in order to evaluate their performance for our use case. With two dataset sizes, we confirmed the superiority of the DeepSMILES over the SMILES representation and continued to use DeepSMILES exclusively.

For our model (Fig. 3), we employed an autoencoderbased network with TensorFlow 2.0 [21] at the backend. This kind of network refers to the model designed by Xu et al. [22], in their work on Show, Attend and Tell, where they demonstrate a higher accuracy for an Image caption generation system with the attention mechanism. The TensorFlow team used these results for their 


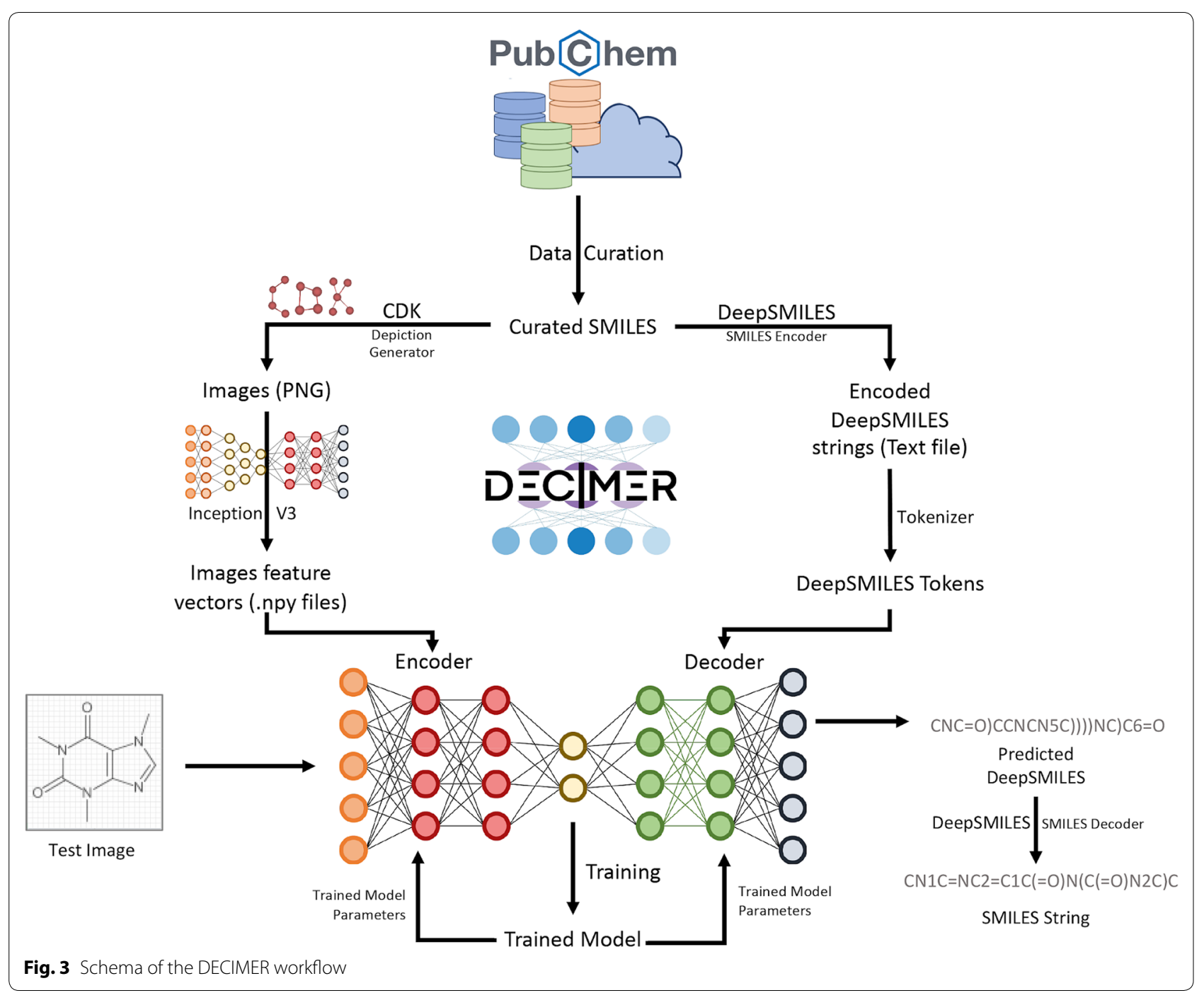

implementation of Show, Attend and Tell, published at [23], which is used unaltered by us. Their encoder network is a convolutional NN (CNN), which consists of a single fully connected layer and a RELU activation function. Their decoder network is a recurrent NN (RNN), consisting of a gated recurrent unit (GRU) and two fully connected layers. The soft attention mechanism used in [23] was introduced by Bahdanau et al. [24].

We trained the model with DeepSMILES textual data and the corresponding bitmap of the chemical diagram. The text file is read by the model, the DeepSMILES is tokenized by the tokenizer, and the unique tokens are stored. The images are converted into feature vectors by using the unaltered Inception V3 [25] model and saved as NumPy arrays.
The model accuracy is determined by the average of all the calculated Tanimoto similarity scores as well as the number of Tanimoto 1.0 hits. The Tanimoto coefficient is superior to simple structure isomorphism because it yields the improvement of the recognition even when identity is not (yet) reached, and with a low degeneracy fingerprint such as the Pubchem fingerprint used here, the Tanimoto 1.0 is almost identical to the more rigorous structure isomorphism.

Initially, we trained multiple models with small training datasets to obtain the best hyper-parameters for our network. Exploration of the hyperparameter space led to 640 images per batch size, with embedding dimension size of 600 for the images, which we depicted on a $299 \times 299$ canvas size to match the Inception V3 model. 
Table 1 Dataset sizes used in this work with corresponding computing times

\begin{tabular}{lrrrr}
\hline Dataset index & Train data size & Test data size & Avrg. time/epoch (s) & $\begin{array}{c}\text { Time for 25 } \\
\text { epochs (s) }\end{array}$ \\
\hline 1 & 54,000 & 6000 & 94.32 & 2358 \\
2 & 90,000 & 10,000 & 159.88 & 3997 \\
3 & 450,000 & 50,000 & 880.6 & 22,015 \\
4 & 900,000 & 100,000 & 2831.8 & 70,795 \\
5 & $1,800,000$ & 200,000 & 7239.28 & 180,982 \\
6 & $2,700,000$ & 300,000 & $11,964.72$ & 299,118 \\
7 & $4,050,000$ & 450,000 & $17,495.12$ & 437,378 \\
8 & $5,850,000$ & 650,000 & 25,702 & 642,550 \\
9 & $7,200,000$ & 800,000 & $32,926.8$ & 823,170 \\
10 & $8,969,751$ & 996,639 & $41,652.24$ & $1,041,306$ \\
11 & $12,600,000$ & $1,400,000$ & $64,909.28$ & $1,622,732$ \\
12 & $15,102,000$ & $1,678,000$ & $91,880.84$ & $2,297,021$ \\
\hline
\end{tabular}

The time for training the model with 15 million structures corresponds to approximately a month on a single Tesla V100 GPU

We used an Adam optimizer with a learning rate 0.0005 and Sparse Categorical Cross entropy to calculate the loss. We trained all the models for 25 epochs, which typically led to convergence. Once the models converged, we started the evaluation of the test set.

The models were trained on an inhouse server equipped with an nVidia Tesla V100 Graphics Card, 384 GB of RAM and two Intel(R) Xeon(R) Gold 6230 CPUs. Even though the training entirely happens on the GPU, the initial dataset preparation was CPU-based.

Training time obviously scales with data size (Table 1, Fig. 4). Model success was evaluated with an independent test data set. During the preparation of this manuscript, initial experiments with parallel training indicated that scaling was not satisfactory beyond 2 or 3 GPUs.

Here we report our results for training data sizes between 54,000 and 15,000,000 structures, with the largest training data set taking 27 days to converge on the hardware reported above (Table 1, Fig. 4). Figures 5 and 6 show the growth of the accuracy of predictions with increasing train data size.

Training success was determined with a number of indicators (Figs. 5 and 6), such as the percentage of Tanimoto 1.0 predictions, the average Tanimoto similarity of all predictions, and the percentage of invalid SMILES produced by the model. Figure $5 \mathrm{a}$ demonstrates that the model's ability to produce valid SMILES and avoid invalid ones steeply increases with larger training datasets. The same can be observed for the two key parameters of this application, the average Tanimoto similarity and the Tanimoto 1.0 percentage, which indicate the fitness of the model to accurately generate a machine-readable structure from a bitmap of a chemical diagram. We show here that the similarity of predicted chemical graphs to the correct chemical graph becomes constantly better with more training data. While we regularly operate with the chemical paradigm that similar structures have similar properties and therefore increasing structure similarity would convey increasing similarity of inherent properties, we chose to confirm that this would hold in our application case. We therefore further evaluated the models' success with additional descriptors such as $\log \mathrm{P}$ or ring count between original and the predicted SMILES, which indicates that the model consistently produces better and better machine representations with growing training data size. The improvements do not seem to converge prematurely.

In order to assess the promise of these preliminary results, we performed an idealistic linear extrapolation of our data toward larger training data sizes, which indicate that close-to-perfect detection of chemical structures would require training data sizes with 50 to 100 million structures. Such a training data volume will likely require a training time of 4 months with our setup with a single GPU. We are currently experimenting with the distributed learning solution currently available in the Tensorflow 2.0 API to reduce this training time significantly, also evaluating Google's Tensor Processing Units (TPU). 


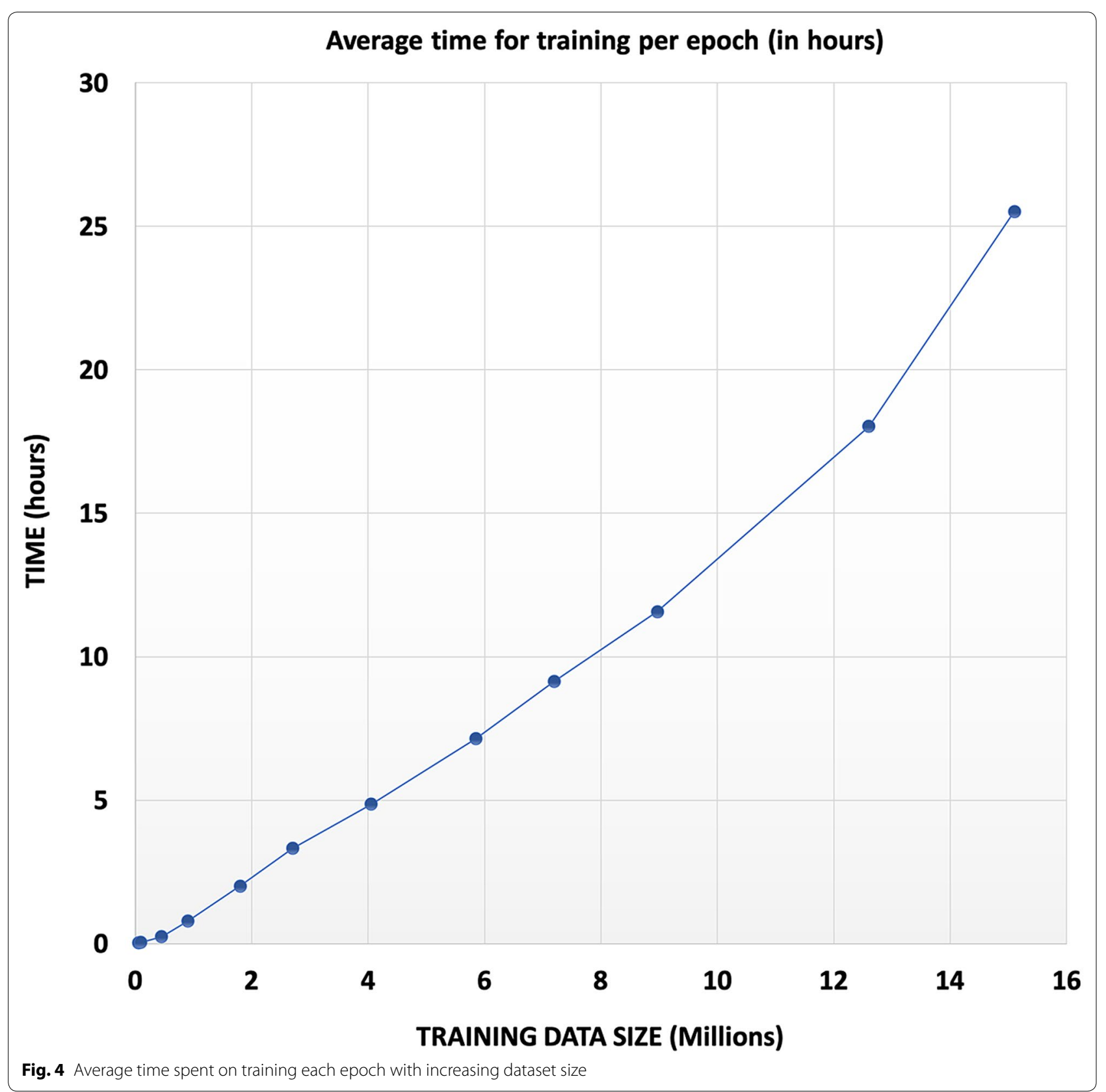

\section{Conclusions}

Here we have presented preliminary results indicating that a show-and-tell deep neural network setup has the potential to successfully extract a machine-readable structure representation when trained with tens of millions of examples. The training setup makes minimal assumptions about the problem. Training success depended on the input data representation. DeepSMILES were superior over SMILES and we have the preliminary indication that the recently reported SELFIES outperform DeepSMILES. For example, for a training data size of 6 Mio images, we obtained an average Tanimoto similarity of 0.53 with DeepSMILES and 0.78 with SELFIES. An extrapolation of our results towards larger training data sizes suggests that we might be able to achieve near-accurate prediction with 50 to 100 million training structures. Such training can be completed in uncomfortable but feasible time spans of several months on a single GPU. 


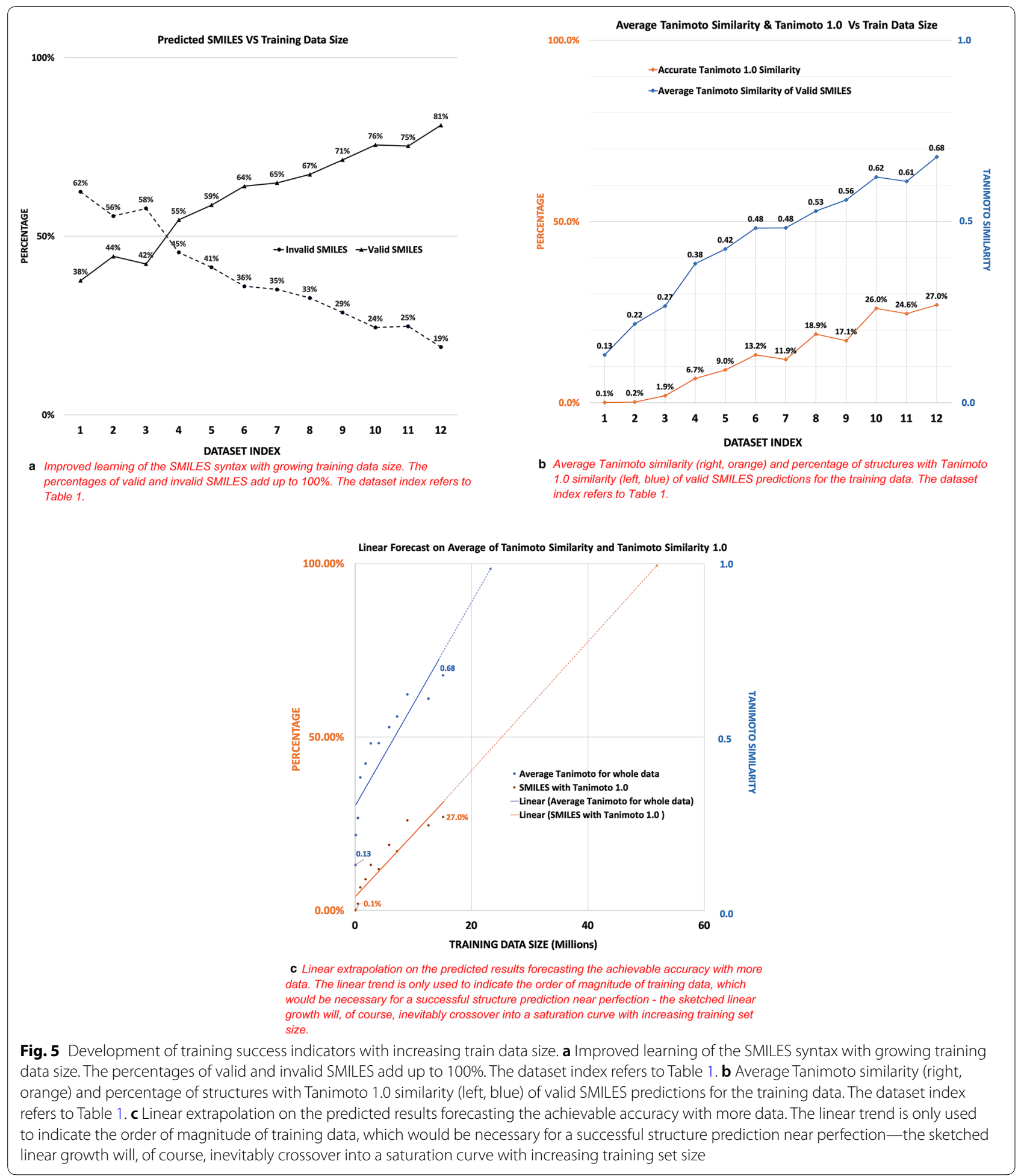

Our work is entirely based on open-source software and open data and is available to the general public for any purpose.
We are currently moving towards larger training sets with the use of parallelization and more powerful hardware and hope to report the results in a full paper on this work in due time. 


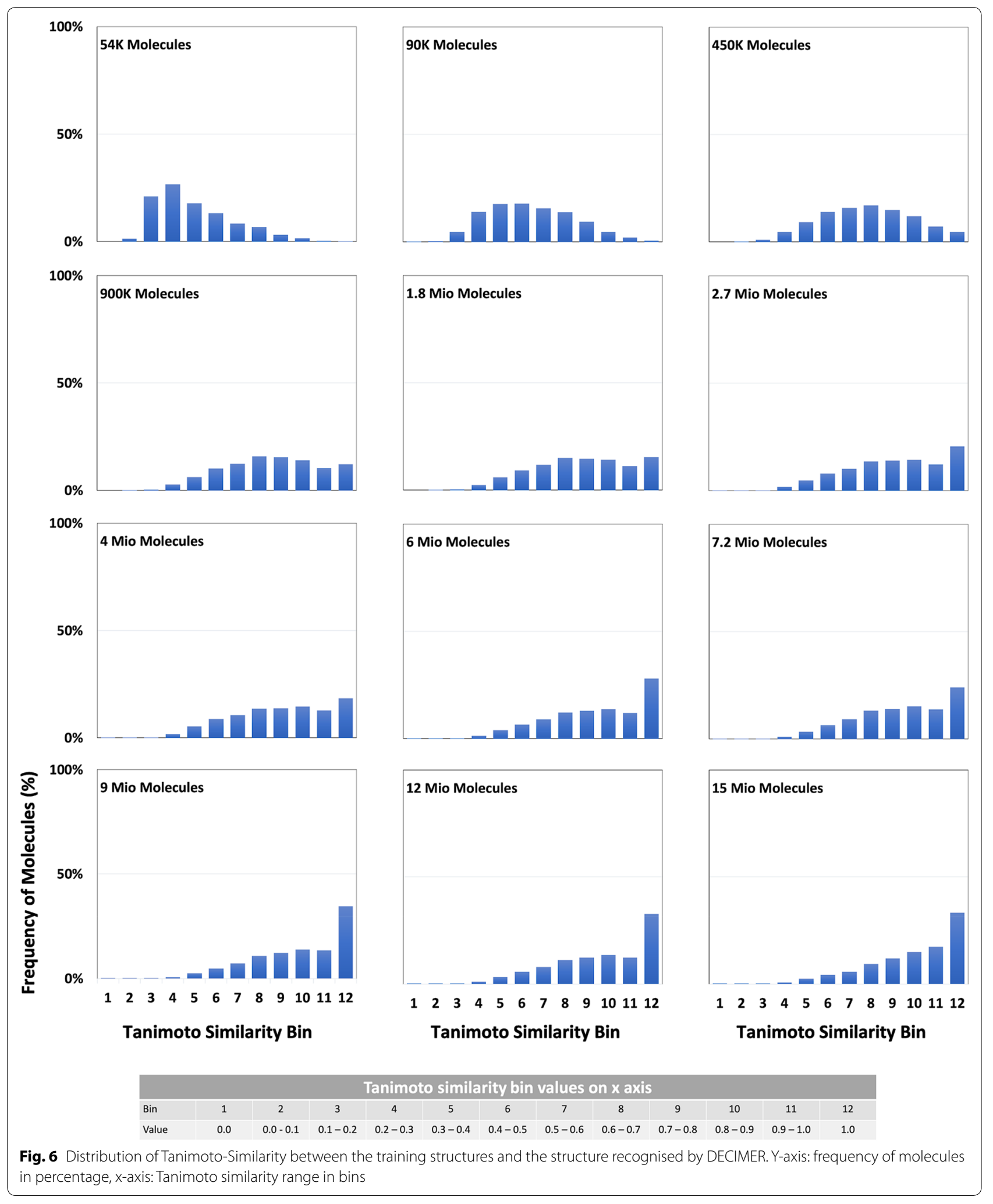




\section{Acknowledgements Not applicable.}

\section{Authors' contributions}

KR developed the software and performed the data analysis. CS and AZ conceived the project and supervised the work. All authors contributed to, read and approved the final manuscript.

\section{Funding}

Open Access funding enabled and organized by Projekt DEAL. The authors acknowledge funding by the Carl-Zeiss-Foundation.

\section{Availability of data and materials}

The code for DECIMER is available at https://github.com/Kohulan/DECIMER, https://github.com/Kohulan/DECIMER-Image-to-SMILES.

\section{Competing interests}

The authors declare that they have no competing interests.

\section{Author details}

${ }^{1}$ Institute for Inorganic and Analytical Chemistry, Friedrich-Schiller-University Jena, Lessingstr. 8, 07743 Jena, Germany. ${ }^{2}$ Institute for Bioinformatics and Chemoinformatics, Westphalian University of Applied Sciences, August-Schmidt-Ring 10, 45665 Recklinghausen, Germany.

\section{Received: 11 June 2020 Accepted: 13 October 2020}

Published online: 27 October 2020

\section{References}

1. McDaniel JR, Balmuth JR (1992) Kekule: OCR-optical chemical (structure) recognition. J Chem Inf Model 32(4):373-378. https://doi.org/10.1021/ ci00008a018

2. Borman S (1992) New computer program reads, interprets chemical structures. Chem Eng News 70(12):17-19. https://doi.org/10.1021/cenv070n012.p017

3. Contreras ML, Allendes C, Alvarez LT, Rozas R (1990) Computational perception and recognition of digitized molecular structures. J Chem Inf Model 30(3):302-307. https://doi.org/10.1021/ci00067a014

4. Casey R, Boyer S, Healey P, Miller A, Oudot B, Zilles K (1993) Optical recognition of chemical graphics. In: Proceedings of 2 nd international conference on document analysis and recognition (ICDAR '93). IEEE Computer Society Press, Washington, DC, pp 627-631. https://ieeexplore.ieee.org/ document/395658/

5. Ibison P, Jacquot M, Kam F, Neville AG, Simpson RW, Tonnelier C et a (1993) Chemical literature data extraction: the CLiDE Project. J Chem Inf Model 33(3):338-344. https://doi.org/10.1021/ci00013a010

6. Zimmermann M, Bui Thi LT, Hofmann M (2005) Combating illiteracy in chemistry: towards computer-based chemical structure reconstruction. ERCIM News 60(60):40-41. https://www.ercim.eu/publication/Ercim _News/enw60/zimmermann.html, https://www.researchgate.net/publi cation/228766116_Combating_illiteracy_in_chemistry_towards_compu ter-based_chemical_structure_reconstruction

7. Algorri M-E, Zimmermann M, Friedrich CM, Akle S, Hofmann-Apitius M (2007) Reconstruction of chemical molecules from images. In: 2007 29th annual international conference of the IEEE engineering in medicine and biology society. IEEE, New York, pp 4609-4612. https://ieeexplore.ieee. org/document/4353366/

8. Algorri M-E, Zimmermann M, Hofmann-Apitius M (2007) Automatic recognition of chemical images. In: Eighth Mexican international conference on current trends in computer science (ENC 2007). IEEE, New York, pp 41-46. https://ieeexplore.ieee.org/document/4351423/

9. Park J, Rosania GR, Shedden KA, Nguyen M, Lyu N, Saitou K (2009) Automated extraction of chemical structure information from digital raster images. Chem Cent J 3(1):4. https://doi.org/10.1186/1752-153X-3-4

10. Filippov IV, Nicklaus MC (2009) Optical structure recognition software to recover chemical information: OSRA, an open source solution. J Chem Inf Model 49(3):740-743. https://doi.org/10.1021/ci800067r
11. Karthikeyan M (2017) Chemical structure recognition tool. US Patent 9,558,403 B2

12. Kwon O-S, Kim D, Kim C-K, Sun J, Sim CJ, Oh D-C et al (2020) CytotoxiC scalarane sesterterpenes from the sponge Hyrtios erectus. Mar Drugs 18(5):253

13. Silver D, Schrittwieser J, Simonyan K, Antonoglou I, Huang A, Guez A et al (2017) Mastering the game of go without human knowledge. Nature 550(7676):354-359. https://doi.org/10.1038/nature24270

14. Staker J, Marshall K, Abel R, McQuaw CM (2019) Molecular structure extraction from documents using deep learning. J Chem Inf Model 59(3):1017-1029

15. Oldenhof M, Arany A, Moreau Y, Simm J (2020) ChemGrapher: optical graph recognition of chemical compounds by deep learning. https:// arxiv.org/abs/2002.09914

16. Willighagen EL, Mayfield JW, Alvarsson J, Berg A, Carlsson L, Jeliazkova N et al (2017) The Chemistry Development Kit (CDK) v2.0: atom typing, depiction, molecular formulas, and substructure searching. J Cheminform 9(1):33. https://doi.org/10.1186/s13321-017-0220-4

17. Kim S, Chen J, Cheng T, Gindulyte A, He J, He S et al (2019) PubChem 2019 update: improved access to chemical data. Nucleic Acids Res 47(D1):D1102-D1109

18. Weininger D (1988) SMILES, a chemical language and information system: 1: introduction to methodology and encoding rules. J Chem Inf Comput Sci 28(1):31-36

19. O'Boyle N, Dalke A (2018) DeepSMILES: an adaptation of SMILES for use in machine-learning of chemical structures. chemRxiv: 1026434, pp 1-9. https://github.com/nextmovesoftware/deepsmiles

20. Krenn M, Häse F, Nigam A, Friederich P, Aspuru-Guzik A (2020) Selfreferencing embedded strings (SELFIES): a 100\% robust molecular string representation. https://github.com/aspuru-guzik-group/selfies. Accessed 2 June 2020

21. Abadi M, Agarwal A, Barham P, Brevdo E, Chen Z, Citro C et al (2016) TensorFlow: large-scale machine learning on heterogeneous distributed systems. https://arxiv.org/abs/1603.04467

22. Xu K, Ba JL, Kiros R, Cho K, Courville A, Salakhutdinov R et al (2015) Show, attend and tell: neural image caption generation with visual attention. In: 32 nd International conference on machine learning, ICML 2015, vol 3, pp 2048-2057

23. tensorflow. tensorflow/docs. https://github.com/tensorflow/docs/blob/ master/site/en/tutorials/text/image_captioning.ipynb. Accessed 18 Aug 2020

24. Bahdanau D, Cho KH, Bengio Y (2015) Neural machine translation by jointly learning to align and translate. In: 3rd international conference on learning representations, ICLR 2015-Conf Track Proc, pp 1-15

25. Szegedy C, Vanhoucke V, loffe S, Shlens J, Wojna Z (2016) Rethinking the inception architecture for computer vision. In: Proceedings of the IEEE computer society conference on computer vision and pattern recognition, pp 2818-2826

\section{Publisher's Note}

Springer Nature remains neutral with regard to jurisdictional claims in published maps and institutional affiliations.

Ready to submit your research? Choose BMC and benefit from

- fast, convenient online submission

- thorough peer review by experienced researchers in your field

- rapid publication on acceptance

- support for research data, including large and complex data types

- gold Open Access which fosters wider collaboration and increased citations

- maximum visibility for your research: over 100M website views per year

At BMC, research is always in progress.

Learn more biomedcentral.com/submissions 